\title{
Suppressor of Cytokine Signaling-3 Suppresses the Ability of Activated Signal Transducer and Activator of Transcription-3 to Stimulate Neurite Growth in Rat Primary Sensory Neurons
}

\author{
Tizong Miao, ${ }^{1 \star}$ Dongsheng Wu, ${ }^{1 \star}$ Yi Zhang, ${ }^{1}$ Xuenong Bo, ${ }^{1}$ Maria Cristina Subang, ${ }^{2}$ Ping Wang, ${ }^{3}$ and \\ Peter M. Richardson ${ }^{1}$ \\ Centres for ${ }^{1}$ Neuroscience, ${ }^{2}$ Bone and Joint, and ${ }^{3}$ Gastroenterology, Barts and the London Queen Mary's School of Medicine, University of London, London \\ E1 2AT, United Kingdom
}

\begin{abstract}
The actions of the neuropoietic cytokines are mediated by the gp130 receptor, which activates several signaling molecules including the transcription factor STAT3 (signal transducer and activator of transcription), which, in turn, is subject to feedback inhibition by SOCS3 (suppressor of cytokine signaling). Activation of the gp130 receptor has been implicated in axonal growth particularly during regeneration, but the specific contribution of STAT3 is the subject of conflicting reports. Measurements of SOCS3 mRNA in rat dorsal root ganglia showed a significant induction in this inhibitory molecule after peripheral nerve injury. The functions of STAT3 and SOCS3 in adult rat primary sensory neurons were investigated in vitro through transduction of lentiviruses yielding a conditionally activated STAT3, native SOCS3, or a mutant SOCS3 with dominant-negative actions. The SOCS3 construct was effective in inhibiting tyrosine phosphorylation of STAT3 in a neuroblastoma cell line and in blocking nuclear accumulation of endogenous STAT3 or of the conditionally activated STAT3 chimera in primary sensory neurons. In such neurons, transduction and activation of STAT3 enhanced neurite growth, transduction with SOCS3 reduced neurite outgrowth, and transduction with mutant SOCS3 enhanced neurite growth, at least under basal conditions. In conclusion, STAT3 signaling is beneficial to axonal growth through activating transcription of unidentified genes, and SOCS3 is detrimental to axonal growth through inhibition of STAT3 and/or other transcription factors.
\end{abstract}

Key words: SOCS3; STAT3; neurite growth; primary sensory neuron; cytokine; conditioning

\section{Introduction}

Axonal growth is regulated by post-transcriptional events at the growth cone (Li et al., 2004; Ren et al., 2004; Zhou et al., 2004), which are modified in ways that are not yet precisely defined by the products of "regeneration-associated genes" (Kobayashi et al., 1997; Bomze et al., 2001). The molecular changes within primary sensory neurons that mediate a propensity for regeneration after peripheral nerve injury are instigated, at least in part, by neuropoietic cytokines such as interleukin-6 (IL-6), leukemia inhibitory factor (LIF), and ciliary neurotrophic factor (CNTF) and by the gp130/Janus kinase (JAK) receptor complex. Evidence for this statement includes observations that the concentration of IL-6 is increased in dorsal root ganglia (DRGs) after nerve injury (Murphy et al., 1995), that regeneration is impaired in mice with null deletion of LIF (Cafferty et al., 2001) or IL-6 (Galiano et al., 2001; Cafferty et al., 2004), and that regenerative responses are

\footnotetext{
Received May 22, 2006; revised Aug. 7, 2006; accepted Aug. 10, 2006

This work was supported by Corporate Action Trust and the Special Trustees of the Royal London Hospital.

*T.M. and D.W. contributed equally to this work.

Correspondence should be addressed to Dr. Peter M. Richardson at the above address. E-mail: p.richardson@qmul.ac.uk.

D01:10.1523/JNEUROSCI.2160-06.2006

Copyright $\odot 2006$ Society for Neuroscience $\quad 0270-6474 / 06 / 269512-08 \$ 15.00 / 0$
}

suppressed by pharmacological inhibition of JAK2 (Liu and Snider, 2001).

gp130 is the unique signaling receptor for IL- 6 and combines with the LIF $\beta$ receptor to mediate the actions of CNTF and LIF. Dimerization of gp130 leads to recruitment and phosphorylation of receptor-associated JAK1 and JAK2, which phosphorylate and cause the dimerization of STAT3 (signal transducer and activator of transcription), leading to its nuclear translocation and the transcription of target genes. Although STAT3 is its major signaling pathway, gp130 also activates extracellular-regulated kinase (ERK), phosphoinositide 3 kinase, and other signaling pathways (Heinrich et al., 2003). The specific contribution to neurite growth of STAT3, as distinct from gp130, is controversial. In pheochromocytoma PC12 cells, results of experiments involving directed mutagenesis of gp130 suggested that STAT3 signaling inhibits differentiation (Ihara et al., 1997), whereas results with a dominant-negative mutant of STAT3 implied the contrary (Wu and Bradshaw, 2000). In both PC12 cells and mouse sympathetic neurons, signaling from the LIF $\beta$ receptor is detrimental to neurite growth (Ng et al., 2003). However, the influence of STAT3 signaling on axonal growth in neurons has not been addressed.

In most cell types, SOCS3 (suppressor of cytokine signaling), one of a family of eight, provides feedback inhibition of STAT3 
signaling through its binding to tyrosine residue 759 (human; residue 757 in mouse) (Fischer et al., 2004) of gp130. Null deletion of SOCS3 in mice causes death in utero (Roberts et al., 2001), deletion of SOCS3 in hematopoietic stem cells causes hypersensitivity to granulocyte colony-stimulating factor and inflammatory disease (Croker et al., 2004), deletion of SOCS3 in macrophages suppresses responses to lipopolysaccharide through disinhibition of IL-6 signaling (Yasukawa et al., 2003), and deletion of SOCS3 in the brain increases sensitivity to leptin and resistance to obesity (Mori et al., 2004). In brief, SOCS3 has noncompensable functions in several cell types including neurons; its function in axonal growth has not been evaluated.

Here, it is shown in rat primary sensory neurons in vitro that overexpression and activation of STAT3 stimulates neurite growth, that overexpression of SOCS3 blocks nuclear translocation of both endogenous and exogenous STAT3 and neurite outgrowth, and that neutralization of endogenous SOCS3 through overexpression of a mutant SOCS3 (mSOCS3) stimulates neurite outgrowth.

\section{Materials and Methods}

Animal surgery. All procedures were performed according to United Kingdom Home Office regulations. Adult rats weighing 200-250 g were anesthetized by halothane inhalation and subjected to transection of the left sciatic nerve at mid-thigh level. Rats were allowed to live for $6 \mathrm{~h}$ to 1 month after nerve injury. After they were killed, ipsilateral and contralateral L4 and L5 DRGs (lumbar DRGs) were removed and either stored directly in liquid nitrogen or kept in ice-cold HBSS for neuron culture.

RNA extraction and cDNA synthesis. For extraction of RNA, frozen DRGs or dissociated neurons washed with PBS were pulverized in liquid nitrogen and homogenized in Trizol reagent (Invitrogen, Paisley, UK). After phenol-chloroform extraction and isopropanol precipitation, total RNA was washed twice with $75 \%$ ethanol, air dried, and stored in water with $0.5 \mathrm{U} / \mu \mathrm{l}$ RNase inhibitor at $-80^{\circ} \mathrm{C}$ until use. Reverse transcription of $1-3 \mu \mathrm{g}$ aliquots of RNA was performed with Superscript III reverse transcriptase (Invitrogen), and the enzyme was inactivated by heating at $70^{\circ} \mathrm{C}$ for $15 \mathrm{~min}$.

Real-time PCR. cDNA synthesized from 50-100 ng of total RNA extracted from L4 and L5 DRGs was used for quantitative real-time PCR in a Rotor-Gene 3000 machine (Corbett Research, Sydney, Australia) with SYBR Green chemistry (Qiagen, Hilden, Germany). To generate standard curves for SOCS genes and 18S RNA, adult rat spleen was harvested $24 \mathrm{~h}$ after intraperitoneal injection of lipopolysaccharide. The primers were $5^{\prime}$-TGGCTACTGCAGTGCACCTG- $3^{\prime}$ and $5^{\prime}$-TCCAGCTGTCACATGCATGC-3' for CIS (cytokine-inducible SH2 domaincontaining protein; genome survey sequence ID (GI): 13277785), $5^{\prime}$-TTGGTGCGCGACAGTCG-3' and 5'-ACGTAGTGCTCCAGCAGCTC-3' for SOCS1 (GI: 22024395), 5'-GACGTCAGCTGGACCGACTAAC-3' and 5'-GGTACATTTGTTAATGGCGAGTCG-3' for SOCS2 (GI: 76780240), 5'-TTCTTCACACTGAGCGTCGAGA-3' and 5'-CTTGAGTACACAGTCAAAGCGGG-3' for SOCS3 (GI: 3319323), 5' -CGAGAGAAGACGGCTTAGTATCGA-3' and 5' -CACGAGGCAGTGTATGTAATCGATC-3' for SOCS5 (GI: 31418506), $5^{\prime}$-TCAAGATGGAGATCGCGAGTG-3' and 5' -CACCTCCATGGGAGAAGACGTAT-3' for SOCS6 (GI: 55154428), 5' -CTTCCCATCACCCCTGTAAA- $3^{\prime}$ and $5^{\prime}$-ACGTTGTTGGGAAATGGTTC-3' for LIF (GI: 3298136), 5' -CAAGACCATCCAACTCATCTTG-3' and 5' -CACAGTGAGGAATGTCCACAAAC-3' for IL-6 (GI: 7549768), and 5'CGGCTACCACATCCAAGGAA-3' and 5'-TGCTGGCACCAGACTTGCCCTC-3' for 18 S (GI: 57149). The reactions were performed in QuantiTect SYBR Green PCR buffer with HotstarTaq DNA polymerase. The PCR program was 1 cycle of $2 \mathrm{~min}$ at $50^{\circ} \mathrm{C}, 1$ cycle of $15 \mathrm{~min}$ at $95^{\circ} \mathrm{C}$, 45 cycles of denaturation for $20 \mathrm{~s}$ at $95^{\circ} \mathrm{C}$, annealing for $30 \mathrm{~s}$ at $55^{\circ} \mathrm{C}$, and extension for $45 \mathrm{~s}$ at $72^{\circ} \mathrm{C}$. Samples were tested in triplicate, and average values of the threshold cycle were used for quantification. The concentration of $18 \mathrm{~S}$ mRNA in each sample was used as an internal control.

In situ hybridization. Mouse SOCS3 was subcloned into pBluescript
SK(II) (Stratagene, La Jolla, CA) RNA expression vector, and the plasmid was linearized. Digoxigenin-labeled cRNA probes were generated by in vitro transcription from $1 \mu \mathrm{g}$ of SOCS3 plasmid, performed with the DIG-RNA labeling kit (Roche Diagnostics, Penzberg, Germany). The digoxigenin-labeled cRNA probes were treated by alkaline hydrolysis to reduce their size to $\sim 200$ bases. Cryostat sections from freshly frozen rat ipsilateral and contralateral DRGs were mounted on the same slide, fixed in paraformaldehyde, dehydrated in ethanols, hybridized overnight at $42^{\circ} \mathrm{C}$, washed to a stringency of $0.2 \times$ SSC at $50^{\circ} \mathrm{C}$, and incubated in 4-nitro blue tetrazolium chloride plus 5-bromo-4-chloro-3-indolylphosphate and levamisole until color product was visible.

Construction of lentivirus vectors. Mouse SOCS3 Image clone (5707539; Geneservice, Cambridge, UK) was cloned into the pcDNA3.1(+) vector. Mouse mSOCS3, carrying a point mutation from phenylalanine to alanine (F25A) in the kinase inhibitory region (Sasaki et al. 2000), was generated by mutagenesis PCR. STAT3ER, a conditionally active form of STAT3 (Matsuda et al., 1999; Milocco et al., 1999), was generated by ligation of mouse STAT3 to the ligand-binding domain of mouse estrogen receptor (ER) modified (G525R) to recognize 4-hydroxy-tamoxifen (4HT) but not endogenous estrogen. Subsequently, green fluorescent protein (GFP) behind an internal ribosome entry site (IRES) was inserted downstream of SOCS3, mSOCS3, or STAT3ER in the pcDNA3.1 $(+)$ vector. SOCS3, mSOCS3, and STAT3ER together with IRES-GFP were digested from pcDNA3.1 $(+)$ vector with enzyme and cloned into the PmeI restriction site of the lentivirus transfer vector pRRL-MCS+ (Ruitenberg et al., 2002). The integrity and orientation of the sequences were confirmed by DNA sequencing. Nonreplicative lentiviruses for GFP, SOCS3, mSOCS3, and STAT3ER were each generated by cotransfection of three plasmids, the viral core packaging construct pCMVdeltaR8.74, the vesicular stomatitis virus $G$ envelope protein vector pMD.G.2, and the transfer vector (pRRL-SOCS3-IRES-GFP, pRRLmSOCS3-IRES-GFP, pRRL-STAT3ER-IRES-GFP, or pRRL-GFP) into human embryonic kidney 293T (HEK 293T) cells via calcium precipitation (Ruitenberg et al., 2002). Virus was concentrated by ultracentrifugation and titered in HEK 293T cells by counting GFP-positive cells. The titers of the virus stocks were $\sim 10^{9}$ transducing units $/ \mathrm{ml}$.

Cell line cultures. Human neuroblastoma SH-SY5Y cells were cultured in a 1:1 mixture of Ham's/F-12 and Eagle's minimal essential medium supplemented with $10 \%(\mathrm{v} / \mathrm{v})$ fetal bovine serum. To examine the expression and function of SOCS3 constructs, SH-SY5Y cells were transduced overnight with lentiviruses to produce SOCS3, mSOCS3, or GFP alone.

Neuron cultures. Adult rat DRGs associated with normal or transected sciatic nerves were dissociated in $0.125 \%$ collagenase type XI (Sigma, St. Louis, MO). Dissociated cells were centrifuged through an albumin cushion to enrich for neurons. For experiments involving mRNA measurement, neurons were grown on poly-L-lysine-coated dishes in N2 medium for 2-20 h. Two techniques of infection by lentivirus were used, each with multiplicity of infection (MOI) of $\sim 500$. In experiments involving SOCS3 or mSOCS3, neurons suspended in N2 medium were infected for $24 \mathrm{~h}$. In experiments involving STAT3ER, lentivirus was added $1 \mathrm{~h}$ after the neurons were plated. In either case, the neurons were maintained in serum-free N2 medium on poly-L-lysine-coated slides in 8 -well chambers at 1000 neurons/well for an additional $20 \mathrm{~h}$ for neurons from DRGs associated with nerve injury or for $40-44 \mathrm{~h}$ for neurons from DRGs associated with uninjured sciatic nerves. The shorter time in vitro was used for conditioned neurons because their neurite outgrowth is accelerated (Smith and Skene, 1997). After culturing for 20-44 h, neurons were fixed and immunostained for $\beta 3$-tubulin and, when appropriate, ER. For quantification analysis, the length of the longest neurite of each neuron was measured with ImageJ software (W. S. Rasband, http:// rsb.info.nih.gov/ij/, 1997-2006). Culture for each group was performed in triplicate or more and for each well, and 200-400 infected neurons were quantified. Median neurite lengths and frequency histograms of neurite lengths in three bins ( $<30 \mu \mathrm{m}, 30-200 \mu \mathrm{m}$, and $>200 \mu \mathrm{m})$ were analyzed.

Immunocytofluorescence. Neurons were fixed with $4 \%$ paraformaldehyde and incubated overnight at $4{ }^{\circ} \mathrm{C}$ with primary antibodies, rabbit anti-py-STAT3 (1:200; Cell Signaling Technology, Beverly, MA), mouse 

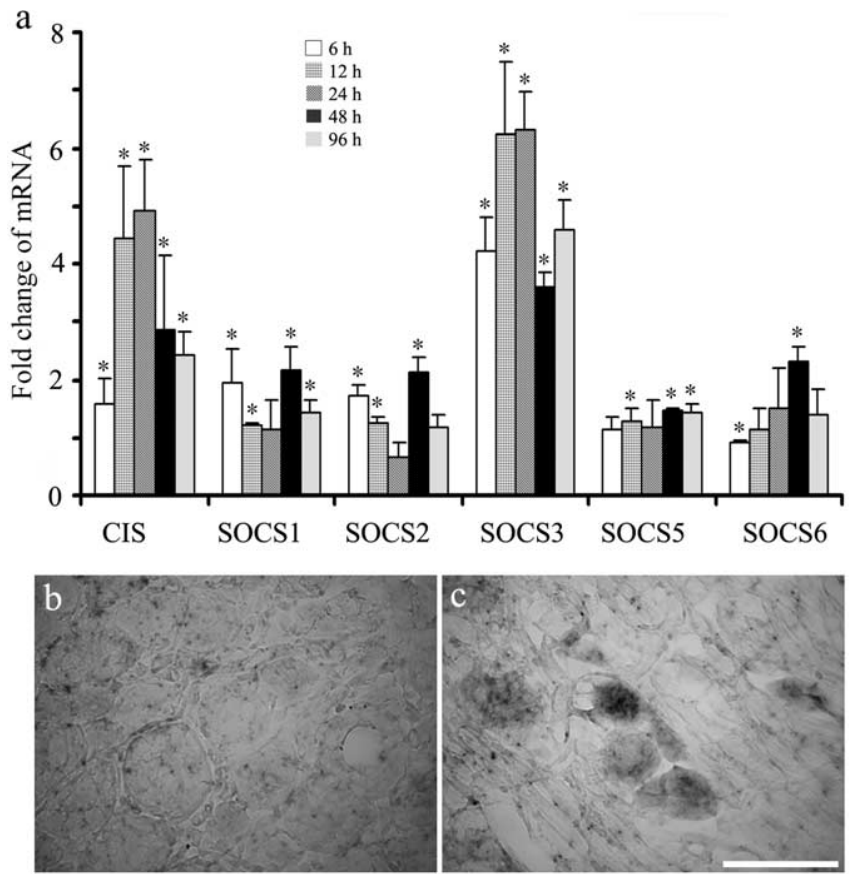

d
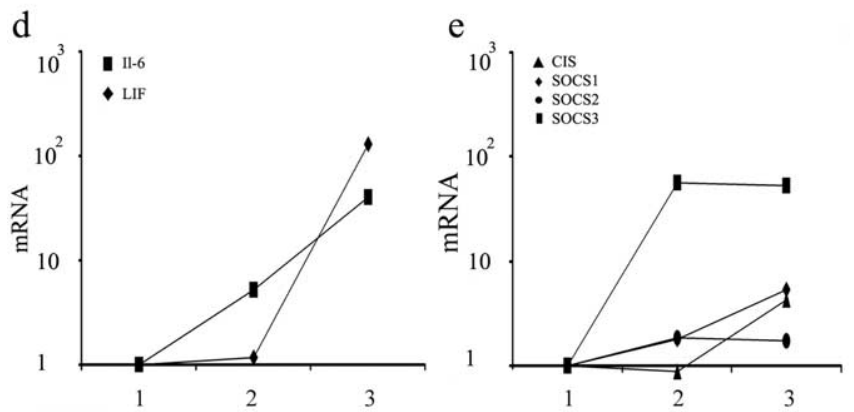

Figure 1. Induction of SOCS3 mRNA in injured neurons. SOCS mRNAs in DRGs removed 6-96 $\mathrm{h}$ after sciatic nerve transection and cultures of DRG cells enriched for neurons are shown. $\boldsymbol{a}$, Real-time PCR measurements. For each time point and each SOCS, the ratio of SOCS mRNA to 18S RNA in DRGs ipsilateral to the sciatic nerve transection was normalized to the ratio in contralateral DRGs (mean \pm SD; $n=3$ animals; ${ }^{*} p<0.05$ vs contralateral DRGs by Student's $t$ test). $\boldsymbol{b}, \boldsymbol{c}$, Photomicrographs of in situ hybridization preparations to illustrate the presence of SOCS3 mRNA in DRG neurons ipsilateral (c) but not contralateral (b) to nerve injury. Scale bar, 50 $\mu \mathrm{m}$. $\boldsymbol{d}, \boldsymbol{e}$, Real-time PCR measurements of SOCS and cytokine mRNAs in DRG neurons after cell dissociation and cell culture. For each condition and each SOCS or cytokine mRNA, the ratio of SOCS mRNA to 18S RNA in DRGs was normalized to the ratio in normal DRGs frozen immediately after removal. 1, Freshly harvested whole DRGs; 2 , cell preparation enriched for neurons several hours later after dissection, dissociation, and centrifugation; 3 , neurons after an additional $20 \mathrm{~h}$ in culture in serum-free medium (means; $n=3$ animals).

anti- $\beta 3$-tubulin (1:400-1:1000; Sigma), or rabbit anti-ER antibody (1: 500; Upstate Technology, Charlottesville, VA) in TBS/T; washed; incubated with secondary antibodies conjugated with tetramethylrhodamine isothiocyanate, fluoroscein isothiocyanate, or 7-amino-3-methylcoumarin acetic acid; and mounted with anti-fade reagent. Images of lentivirus-infected neurons were captured for analysis by NIH ImageJ software.

Western blot analysis. Lysates from human neuroblastoma SH-SY5Y cells were subjected to SDS-PAGE. The proteins were transferred to polyvinylidene difluoride membranes, which were incubated with primary antibodies to phosphorylated tyrosine-704 STAT3, STAT3, phosphorylated ERK, and ERK (all from Cell Signaling Technology) and then with the appropriate horseradish peroxidase-conjugated secondary antibody (Amersham Biosciences, Buckinghamshire, UK). Bands were visualized by enhanced chemiluminescence and quantified with NIH ImageJ software.
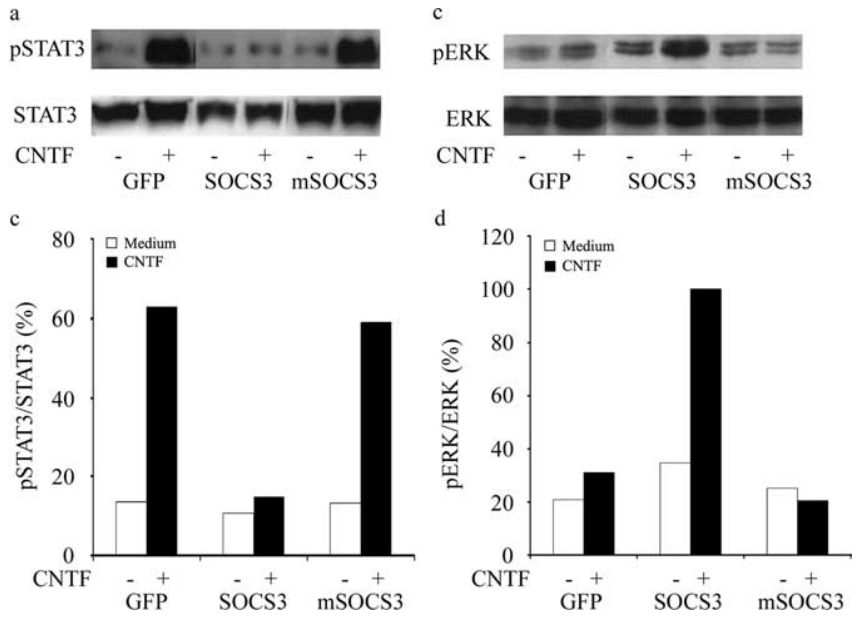

Figure 2. Influence of $\mathrm{SOCS} 3$ and mSOCS3 on signal transduction in SH-SY5Y neuroblastoma cells. $\boldsymbol{a}$, Western blot analysis of STAT3 and tyrosine-phosphorylated STAT3 (pSTAT3). Cells were transduced with LV-GFP, LV-SOCS3, or LV-mSOCS3, maintained in serum-free medium, and subsequently stimulated by $20 \mathrm{pm}$ CNTF for $30 \mathrm{~min}$. Total cell lysates were subjected to SDS-PAGE, and membranes were processed with antibodies to pySTAT3 or STAT3. $\boldsymbol{b}$, Ratio of phosphorylated STAT3 signal to total STAT3 signal. Open bars, Without CNTF; filled bars, with CNTF. c, Western blot analysis of phosphorylated ERK (pERK) and ERK in cells treated as in $\boldsymbol{a} . \boldsymbol{d}$, Ratio of phosphorylated ERK signal to ERK signal. Open bars, Without CNTF; filled bars, with CNTF.

Statistics. Student's $t$ test was used to compare means, logarithmic regression analysis by least squares was used to establish correlations, and $\chi^{2}$ test was used to compare distributions in frequency histograms.

\section{Results}

\section{Presence of SOCS mRNAs in injured neurons in vivo and in} neuronal cell cultures

In extracts from normal rat DRGs, mRNAs for CIS and SOCS1-7 were detected by reverse transcription-PCR (data not shown). SOCS4 and SOCS7 were not studied further because their function is unknown. After sciatic nerve transection, CIS and SOCS3 mRNAs increased substantially (Fig. 1a). For both mRNAs, the increase was significant at all time points measured between 6 and $96 \mathrm{~h}$, although the change in CIS appeared to peak at $12 \mathrm{~h}$ whereas the change in SOCS3 persisted for at least 1 month (data not shown). SOCS1, SOCS2, and SOCS5 mRNAs also increased, in some cases, significantly but less dramatically. In situ hybridization in DRGs associated with sciatic nerves that had been transected $1 \mathrm{~d}$ previously (Fig. $1 \mathrm{c}$ ) demonstrated the presence of SOCS3 mRNA within many large- and medium-sized neurons. A reaction product was not seen in DRGs associated with uninjured sciatic nerves (Fig. 1b) nor in sections reacted with sense mRNA (data not shown). The results indicate that the concentration of SOCS3 mRNA within DRG neurons increases considerably after nerve injury.

mRNAs for CIS, SOCS1, SOCS2, and SOCS3 were also assayed in preparations of cells dissociated from DRGs, enriched for neurons, and maintained in vitro (Fig. 1e) and were compared with values in whole DRGs frozen immediately after removal. SOCS3 mRNA increased $\sim 50$-fold within $5 \mathrm{~h}$ after removal of DRGs and plating of cells and remained at a similarly high concentration after an additional $18 \mathrm{~h}$ in culture. mRNAs for CIS and SOCS1 also increased, although more slowly and to a lesser extent than did SOCS3 mRNA. For comparison, mRNAs for LIF, IL-6, and CNTF were also measured in the same cell preparations (Fig. 1d). Concentrations of IL-6 and LIF mRNAs but not CNTF increased to a similar order of magnitude as did that of SOCS3 

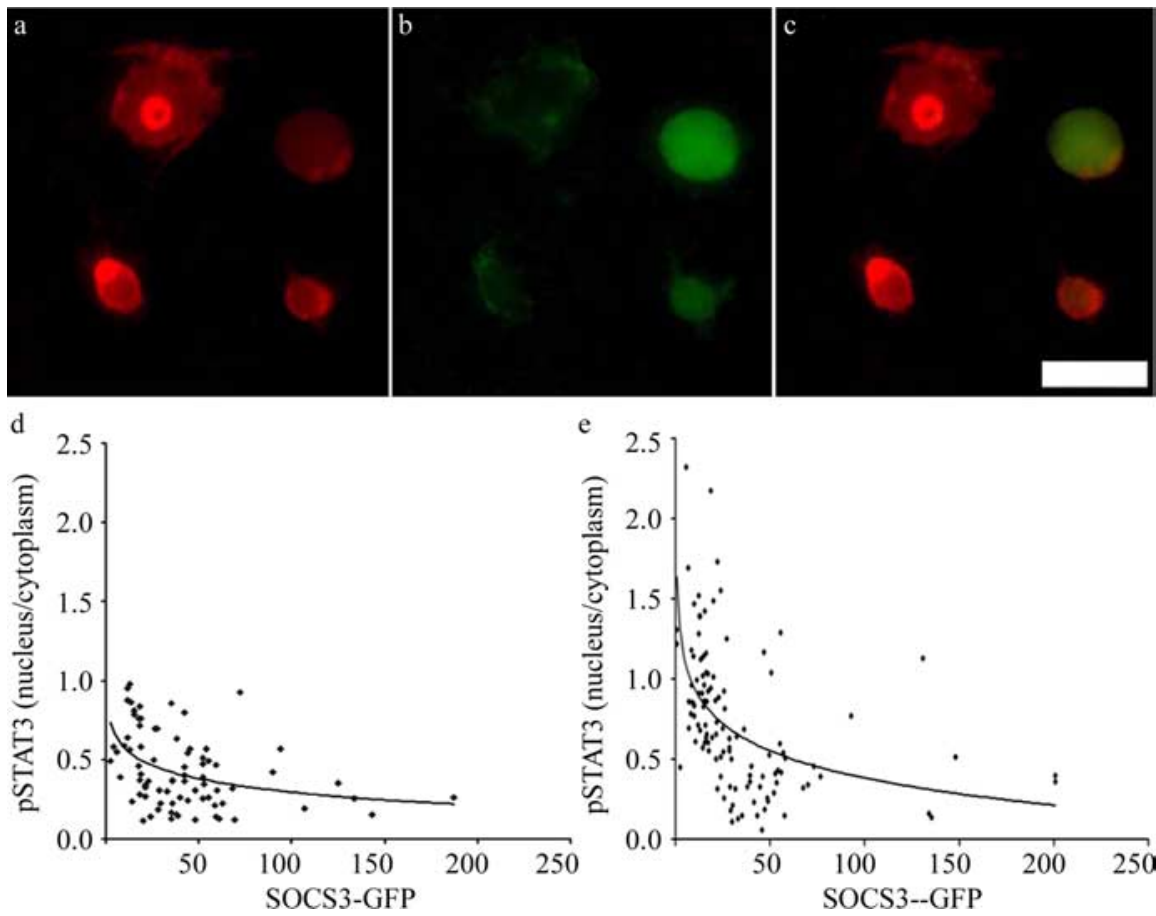

Figure 3. Influence of excess SOCS3 on STAT 3 phosphorylation in DRG neurons. Adult rat DRG neurons were infected by lentivirus constructs for $24 \mathrm{~h}$, cultured for another $48 \mathrm{~h}$, and processed for immunocytochemistry. $\boldsymbol{a}$, Tetramethylrhodamine isothiocyanate immunocytochemistry with antibody to tyrosine-phosphorylated STAT3. $\boldsymbol{b}$, GFP fluorescence to detect neurons successfully transduced with the SOCS3-GFP construct. c, Merged image of $\boldsymbol{a}$ and $\boldsymbol{b}$. Note that the two neurons with GFP have no pySTAT3 immunoreactivity in their nuclei, whereas the two neurons without GFP have strong pySTAT3 immunoreactivity in their nuclei. d, Quantification of pySTAT3 immunoreactivity in cells transduced with SOCS3-GFP. For each neuron, the ratio of nuclear to cytoplasmic pySTAT3 immunoreactivity is plotted against the intensity of GFP fluorescence. The curve fits pySTAT measurement versus $\ln$ (GFP measurement) by least squares ( $r=0.4 ; p<0.01$ that the two measurements are correlated). $\boldsymbol{e}$, As for $\boldsymbol{d}$, except that cells were stimulated by $2 \mathrm{~nm}$ CNTF for 40 min before fixation. The curve fits pySTAT measurement vs In (GFP measurement) by least squares ( $r=0.5 ; p<0.01$ that the two measurements are correlated). Scale bar, $50 \mu \mathrm{m}$.

mRNA, although the induction of the gp130 ligand mRNAs was, if anything, slower than that of SOCS3 mRNA. The results show that mRNAs for SOCS3, IL-6, and LIF appear rapidly in preparations of DRG neurons used for cell culture. The latter two may help to maintain survival and neurite growth of neurons in serum-free medium.

\section{Actions of SOCS3 and mSOCS3 in neuroblastoma cells}

Two lentivirus constructs were prepared to deliver either naive SOCS3 or mSOCS3, mutant SOCS3 inactivated by a phenylalanine-to-alanine mutation at amino acid 25 in the kinase inhibitory domain, each linked to GFP through an IRES, and their efficacy was tested in SH-SY5Y neuroblastoma cells. As judged by green fluorescence, $\sim 95 \%$ of cells were successfully transduced by either construct. By real-time PCR, the concentration of SOCS3 mRNA induced by the virus was $>1000$-fold greater than the endogenous concentration as measured with primers straddling the translation start site (data not shown). Western blots were performed to confirm the expression of SOCS3 proteins (data not shown) and to analyze the influence of the constructs on tyrosine phosphorylation of STAT3 in response to 20 pM CNTF for 30 min (Fig. 2a,b). CNTF-mediated STAT3 phosphorylation was inhibited by lentivirus vector (LV)-SOCS3 and unaltered by LV-mSOCS3. In confirmation of previous evidence that SOCS3 activates Ras (Cacalano et al. 2001), both basal and post-CNTF levels of ERK phosphorylation were greater in cells transduced with LV-SOCS3 than in cells transduced with LV-GFP (Fig. 2c,d). Furthermore, LV-mSOCS3 was shown to inhibit the phosphorylation of ERK induced by CNTF. These observations show that SOCS3 and mSOCS3 modify STAT3 and ERK signaling in the SH-SY5Y neuroblastoma cell line.

\section{Reduction by SOCS3 of phosphorylated} STAT3 in neuronal nuclei

In adult DRG neurons in vitro, transduction rates for LV-SOCS3 and LV-mSOCS3 at an MOI of 500 were $20-30 \%$ as judged by the presence of GFP. After cells had been maintained in culture in the presence of LV-GFP, LV-SOCS3, or LVmSOCS3 for $24 \mathrm{~h}$, the influence of SOCS3 on the nuclear content of tyrosinephosphorylated STAT3 was assayed by immunocytochemistry (Fig. 3) under basal conditions or $45 \mathrm{~min}$ after the addition of IL-6 or CNTF. Because exogenous IL-6 had no effect beyond that of endogenous IL-6, the results with no exogenous gp 130 ligand or with IL- 6 were pooled. The ratio of nuclear to cytoplasmic phosphorylated STAT3 tended to decrease with an increasing amount of GFP fluorescence, and no cell with strong green fluorescence had a high ratio. CNTF increased the mean ratio of nuclear to cytoplasmic phosphorylated STAT3, and, in this group also, cells with strong GFP fluorescence had low nuclearto-cytoplasmic ratios. In other experiments, the nuclear-to-cytoplasmic ratio of phosphorylated STAT3 immunureactivity was found to be $0.55 \pm 0.30$ (mean $\pm \mathrm{SD}$ ) in GFP-transduced neurons and $0.42 \pm 0.22$ in SOCS3-tranduced neurons: the difference was significant at the 0.01 level by the Student's $t$ test. From these observations, it was concluded that STAT3 can be phosphorylated and translocated to the nucleus in a subset of DRG neurons and that this process is blocked by exogenous SOCS3.

\section{Conditional nuclear translocation of STAT3 and its inhibition by SOCS3}

When DRG neurons were infected in vitro with lentiviruses bearing STAT3ER and GFP or GFP alone at an MOI of 500, the efficiency of infection was $\sim 20-30 \%$ (data not shown). In neurons infected with LV-STAT3ER, ER immunoreactivity was detected readily, but GFP expression was relatively weak, demonstrable by immunostaining but not by native fluorescence. In the STAT3ER construct STAT3 was fused to the ligand-binding domain of the ER modified at a single amino acid residue so as to recognize $4 \mathrm{HT}$ but not estrogen. The effectiveness of this construct after transduction into neurons was confirmed by assessing nuclear translocation of STAT3ER after stimulation of 4HT (Fig. 4). Without $4 \mathrm{HT}$ stimulation, nuclear ER immunoreactivity was found in 2\% of ER-immunoreactive neurons. After stimulation with $0.5 \mu \mathrm{M} 4 \mathrm{HT}$ for $40 \mathrm{~h}$, nuclear translocation of STAT3ER was seen in $46 \%$ of ER-positive neurons. Other DRG neurons were transduced with both STAT3ER and SOCS3. In neurons with these two constructs, we were surprised to find that administration of 4HT failed to induce nuclear translocation of STAT3ER. 
From these results, it was concluded that the STAT3ER construct effectively provided inducible nuclear translocation of this transcription factor and that SOCS3 blocked the nuclear translocation of STAT3ER.

\section{Enhancement of neurite outgrowth by STAT3}

To assess the influence of STAT3 on neurite outgrowth, DRG neurons were plated on a poly-L-lysine substratum, infected with lentivirus to deliver GFP or STAT3ER, and cultured in the presence or absence of $4 \mathrm{HT}$ for a total of $40 \mathrm{~h}$. Neurons then were fixed, and the length of the longest neurite was recorded for each ERimmunoreactive neuron (Fig. 5). In neurons transduced with LV-GFP, median neurite lengths in the absence or presence of $4 \mathrm{HT}$ were 26 and $21 \mu \mathrm{m}$. In neurons transduced with LV-STAT3ER, median neurite lengths in the absence or presence of $4 \mathrm{HT}$ were 53 and $86 \mu \mathrm{m}$. The distribution of neurite lengths for neurons transduced with LV-STAT3ER and stimulated by 4 HT differed with very high significance by the $\chi^{2}$ test from that for neurons transduced with LV-STAT3ER but not treated with $4 \mathrm{HT}$ and from that for neurons transduced with GFP alone. The mere presence of STAT3ER, without activation by $4 \mathrm{HT}$, also sufficed to increase neurite length significantly, albeit to a lesser extent. Of other neurons infected with both LV-SOCS3 and LV-STAT3ER, only 10\% had neurites longer than $30 \mu \mathrm{m}$, even after $4 \mathrm{HT}$ administration. These results indicate that activation of STAT3 signaling in DRG neurons increases neurite growth but that the effects of direct dimerization of STAT3 are blocked by SOCS3.

\section{Inhibition of neurite outgrowth by} SOCS3 and enhancement by mSOCS3 The effects of LV-SOCS3 and LVmSOCS3 on neurite outgrowth were then evaluated. Two assays were performed with neurons from DRGs associated with normal sciatic nerves (unconditioned) or nerves that had been transected $3 \mathrm{~d}$ previously (conditioned). In either assay, neurons were dissociated and maintained in suspension for $24 \mathrm{~h}$ in the presence of one of the three lentivirus constructs and plated on poly-L-lysine-coated wells. Neurons taken from DRGs with or without conditioning were maintained for an additional 20 or $44 \mathrm{~h}$, respectively. They were then processed for $\beta 3$-tubulin immunocytochemistry, and neurite lengths were recorded for all green fluorescent neurons that were distinguishable from their neighbors (Fig. 6). For conditioned neurons (Fig. 6d,f), LVSOCS3 decreased the median neurite length from $116 \mu \mathrm{m}$ to 0 $\mu \mathrm{m}$, and LV-mSOCS3 increased the median length to $162 \mu \mathrm{m}$. Compared with LV-GFP-transduced neurons, the changes in
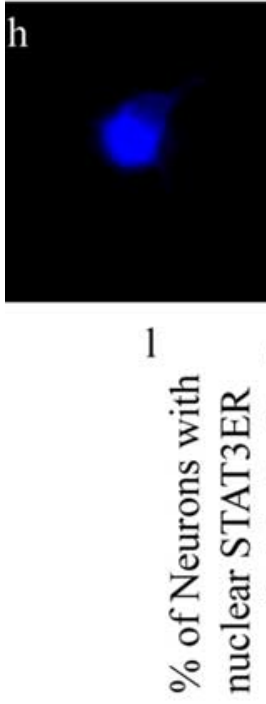
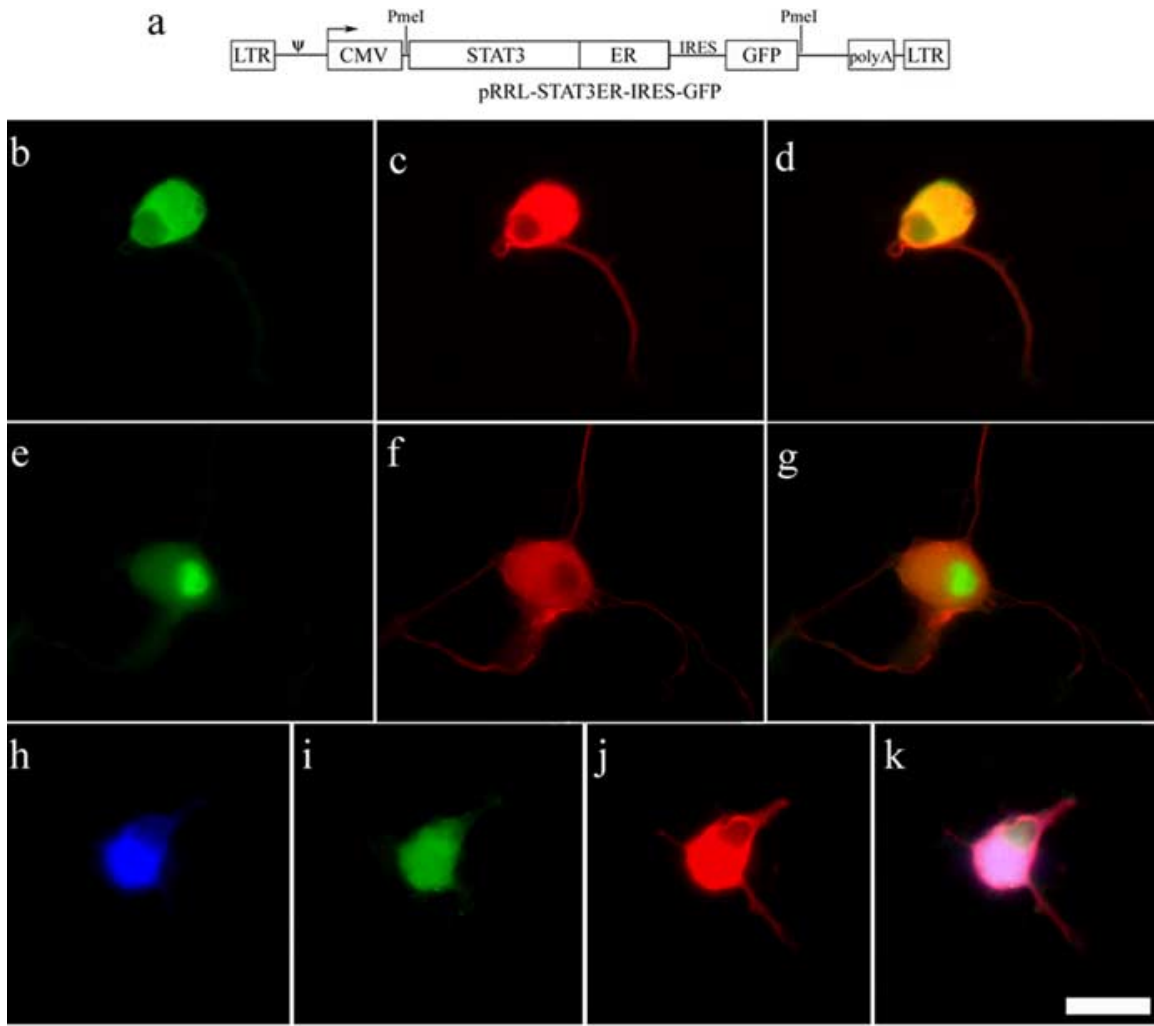

60

Figure 4. Nuclear translocation of the STAT3ER complex in response to 4HT. $\boldsymbol{a}$, Characterization of the plasmid construct, which was cloned into the LV. Components include long terminal repeats (LTR), the $\psi$ encapsidation signal, cytomegalic virus (CMV) promoter, STAT3, modified ligand binding site of ER, IRES, GFP, and polyadenylation tail. $\boldsymbol{b}-\boldsymbol{d}$, Cell transduced with STAT3ER. Green fluorescence $(\boldsymbol{b})$ reflects ER immunoreactivity (used to visualize STAT3ER), and red fluorescence $(\boldsymbol{c})$ reflects $\beta 3$-tubulin immunoreactivity (used to visualize neurons). The images are merged in $\boldsymbol{d}$. Without stimulation of $4 \mathrm{HT}$, ER immunoreactivity is absent from the nucleus, although abundant in the cytoplasm. $\mathbf{e}-\boldsymbol{g}$, As for $\boldsymbol{b}-\boldsymbol{d}$, except that $4 \mathrm{HT}$ has been added for $40 \mathrm{~h}$. Note that this neuron has ER immunoreactivity in the nucleus. $\boldsymbol{h}-\boldsymbol{k}$, A cell cotransduced with STAT3ER (blue fluorescence; $\boldsymbol{h}$ ) and SOCS3/GFP (GFP fluorescence; $\boldsymbol{i}$ ) and immunostained for $\beta 3$-tubulin (red fluorescence; $\boldsymbol{j}$ ). The merged image is in $\boldsymbol{k}$. Note the absence of STAT3ER in the nucleus despite the addition of 4 HT. I, Quantification of nuclear translocation of STAT3ER in DRG neurons in response to $4 \mathrm{HT}$ stimulation (means \pm SEM; $n=4$ cultures; ${ }^{*} p=5 \times 10^{-6}$ by Student's $t$ test). Scale bar, $25 \mu \mathrm{m}$.

median and distribution of neurite lengths of conditioned neurons infected by LV-SOCS3 were highly significant, but those for LV-mSOCS3 were not. For unconditioned neurons transduced with LV-GFP, LV-SOCS3, and LV-mSOCS3 (Fig. 6e,g), median neurite lengths were 0,0 , and $35 \mu \mathrm{m}$, respectively. The differences in distribution of neurite lengths of unconditioned neurons were significant for both LV-SOCS3 and LV-mSOCS3 compared with LV-GFP. Although the median neurite length was 0 , there were always some neurons with neurites (Fig. $6 d, e$ ) and no morphological indication that neurons without neurites were otherwise unhealthy. The results of these experiments indicate that exoge- 


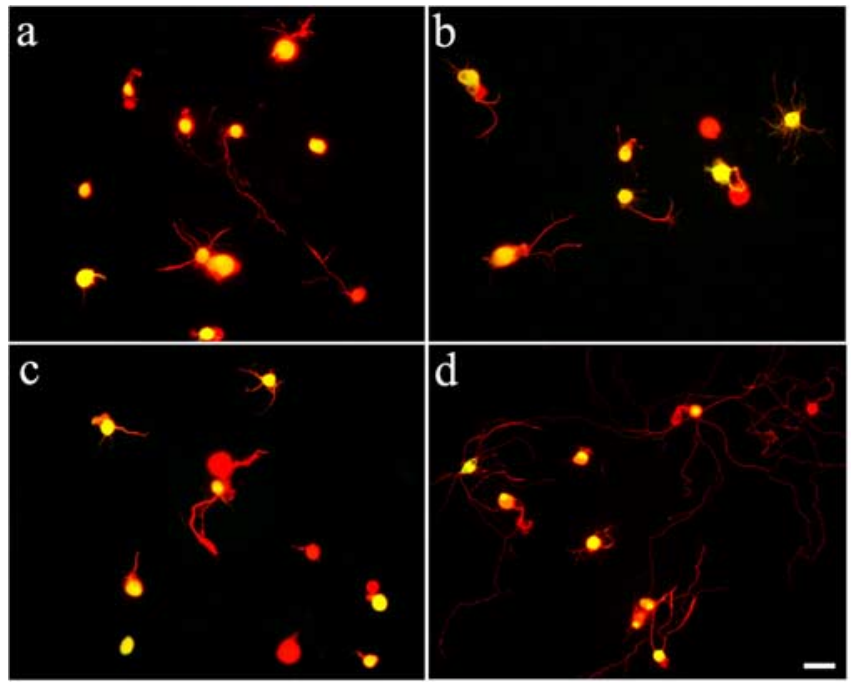

$\mathrm{e}$

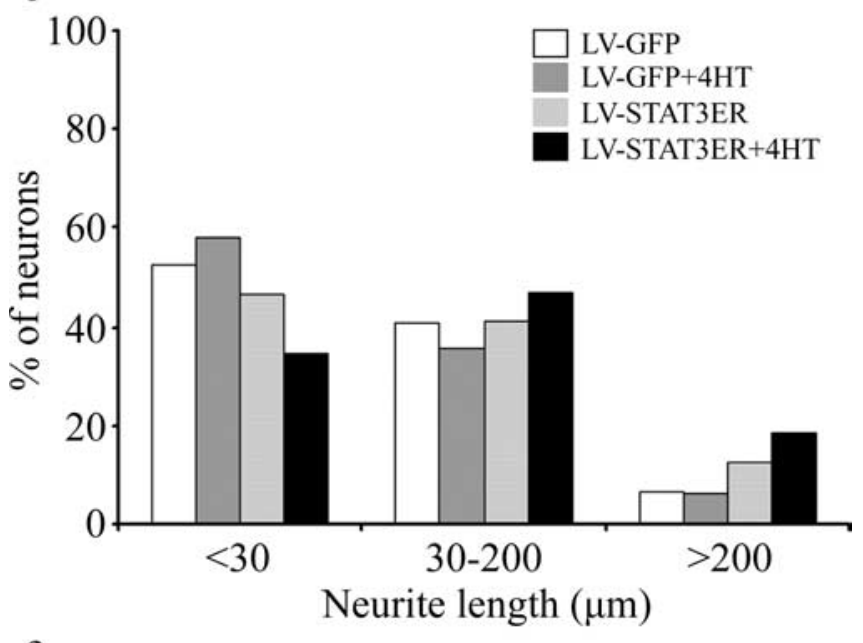

f

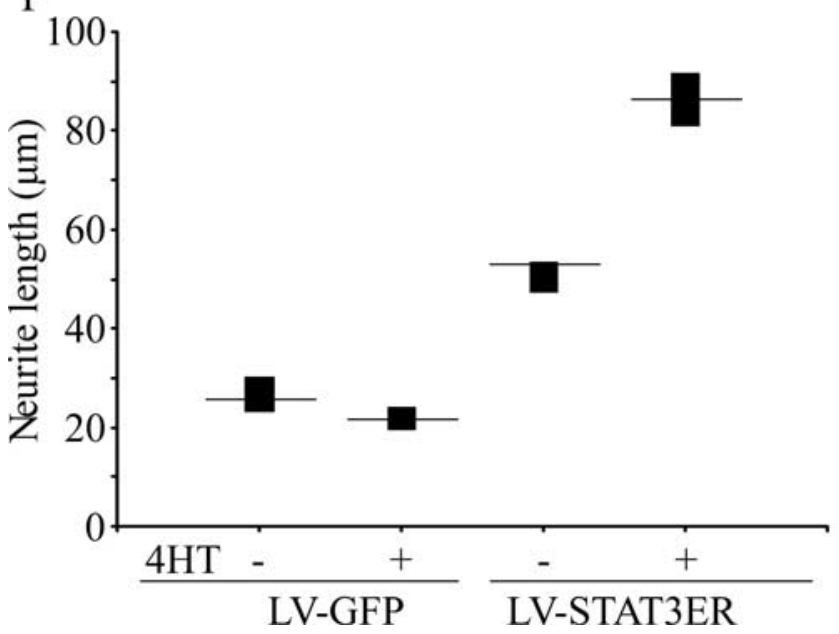

Figure 5. Influence of activation of STAT3 on neurite outgrowth in rat DRG neurons. $\boldsymbol{a}-\boldsymbol{d}$ Neurons were infected with LV-GFP $(\boldsymbol{a}, \boldsymbol{c})$ or LV-STAT3-ER $(\boldsymbol{b}, \boldsymbol{d})$ and cultured for $40 \mathrm{~h}$ in the absence $(\boldsymbol{a}, \boldsymbol{b})$ or presence $(\boldsymbol{c}, \boldsymbol{d})$ of $4 \mathrm{HT}$. $\beta 3$-Tubulin immunoreactivity (red) is used to detect neurons, GFP fluorescence is used to detect LV-GFP-transduced cells, and ER immunoreactivity (green, FITC) is used to detect LV-STAT3ER-transduced cells. Note that neurite outgrowth is enhanced by the activated STAT3ER complex. $e$, Frequency histogram to show the percentage of neurons with neurites in each of the three length ranges ( $n=1840-3471$ neurons per group; $p=5 \times 10^{-18}$, STAT3ER without 4HT vs STAT3ER with 4HT by $\chi^{2}$ analysis). $f$, Neurite lengths in four groups of neurons transduced with GFP or with STAT3ER and with or without 4HT. Median and 95\% confidence levels are plotted. Scale bar, $50 \mu \mathrm{m}$. nous SOCS3 inhibits neurite growth and that counteracting of endogenous SOCS3 can enhance neurite outgrowth, at least in unconditioned neurons.

\section{Discussion}

\section{Influence of STAT3 on neurite outgrowth}

The results show that STAT3 signaling has a beneficial effect on neurite growth. Mere transduction of STAT3ER is sufficient to increase neurite growth beyond baseline levels, and activation by $4 \mathrm{HT}$ results in a highly significant further outgrowth of neurites. We attribute the influence of nonactivated STAT3ER to spontaneous dimerization and nuclear translocation of the unphysiologically high concentration of the molecule. STAT3ER is seen in $2 \%$ of neuronal nuclei at any one time but may influence many more neurons if its stay in the nucleus is short relative to the consequences of its transcriptional activity. The influence of signaling through the chimeric STAT3ER complex may be underestimated because of baseline STAT3 signaling, manifest by the presence of phosphorylated STAT3 in the nuclei of neurons (Fig. $3 d$ ) and presumably stimulated by IL- 6 and LIF in the culture medium (Fig. 1c). The general effect of STAT3 on neurite growth is unequivocal.

It seems a reasonable assumption that STAT3 stimulates neurite outgrowth by modifying the transcription of genes (Dauer et al., 2005) with products that are transported to the growth cone to influence growth-promoting events. One of several candidate proteins is small proline-rich protein $1 \mathrm{~A}$, which is induced by gp130 cytokines (Pradervand et al., 2004) and associates with actin in growth cones (Bonilla et al., 2002). However, the molecular mechanisms by which STAT3 promotes neurite outgrowth remain undefined.

\section{Effects of exogenous SOCS3}

Another conclusion of this study is that exogenous SOCS3 can inhibit neurite outgrowth in primary sensory neurons. The inhibition is highly significant but partial either because SOCS3 is active in only a subpopulation of neurons or because it is partially active in all neurons. One possibility for the incomplete inhibition is that the molecular events that lead to axonal growth have been substantially initiated before effective concentrations of SOCS3 are achieved by infection of cultured neurons with an LV. Although STAT3 favors neurite growth and SOCS3 blocks phosphorylation and nuclear translocation of STAT3 (Fig. 5), it cannot be assumed that inhibition of STAT3 signaling is the only or major mechanism by which SOCS3 blocks neurite outgrowth. In addition to interfering with gp130/JAK/STAT3 signaling, SOCS3 interacts with other signaling molecules such as growth hormone receptor (Greenhalgh and Alexander, 2004), the insulin receptor (Ueki et al., 2004), focal adhesion kinases (Liu et al., 2003), src family kinases (Sitko et al., 2004), NFAT (nuclear factor of activated T cells) (Banerjee et al., 2002), and gp120 rasGAP (Cacalano et al., 2001), all of which could be implicated in the lengthening and branching of neurites.

Because studies on the actions of SOCS3 have focused on its interactions with gp130 and JAKs, our assumption in experiments with cotransduction with SOCS3 and STAT3ER was that SOCS3 would not interfere with downstream events such as dimerization and nuclear translocation of the chimeric molecule. In fact, the results indicate an inhibitory influence of SOCS3 on STAT3 itself. Although we know of no evidence that SOCS3 interacts directly with STAT3, SOCS3 does mediate ubiquitination and degradation of insulin receptor substrates 1 and 2 (Rui et al., 2002). The present evidence for a direct blocking action of SOCS3 

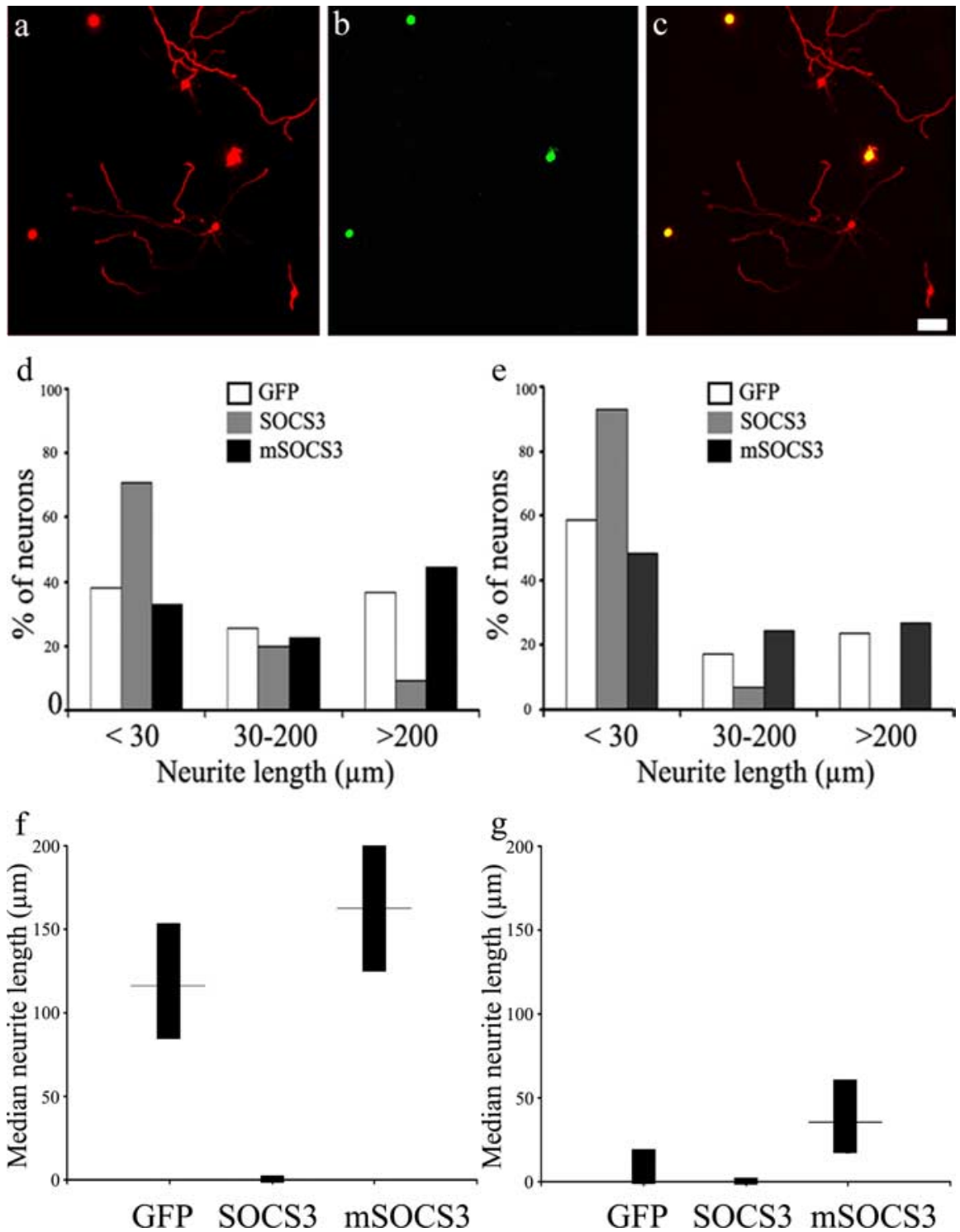

Figure 6. Influence of SOCS3 and mSOCS3 on neurite outgrowth in rat DRG neurons. In $\boldsymbol{a}-\boldsymbol{d}$ and $\boldsymbol{f}$, neurons from DRGs ipsilateral to the side of sciatic nerve transection $3 \mathrm{~d}$ previously were infected by lentivirus for $24 \mathrm{~h}$ and cultured for an additional $20 \mathrm{~h}$. In $\mathbf{e}$ and $\boldsymbol{g}$, neurons from DRGs associated with an intact sciatic nerve were infected by lentivirus for $24 \mathrm{~h}$ and cultured for an additional 44 h. $\boldsymbol{a}$, Immunocytochemistry for $\beta 3$-tubulin (red). $\boldsymbol{b}$, GFP fluorescence to detect neurons successfully transduced with LV-SOCS3-GFP. c, Merged images of $\boldsymbol{a}$ and $\boldsymbol{b}$. Note the paucity of neurites in neurons transduced with SOCS3-GFP. $\boldsymbol{d}$, Frequency histogram for conditioned neurons showing percentages with neurite length in each of three given ranges $(n=219-425$ neurons per group; $p=1 \times 10^{-16}$, SOCS3 vs GFP by $\chi^{2}$ analysis). $e$, Frequency histogram for unconditioned neurons showing percentages with neurite length in each of three given ranges ( $n=325-410$ neurons per group; $p=6 \times 10^{-27}$, SOCS3 vs GFP by $\chi^{2}$ analysis; $p=0.01, \mathrm{mSOCS3}$ vs GFP by $\chi^{2}$ analysis). $\boldsymbol{f}, \boldsymbol{g}$, Neurite lengths for conditioned $(\boldsymbol{f})$ and unconditioned $(\boldsymbol{g})$ neurons transduced with GFP, SOCS3, or mSOCS3. Median lengths and 95\% confidence levels are plotted. Scale bar, $100 \mu \mathrm{m}$.

on STAT3 indicates a novel mechanism of action of SOCS3 in the STAT3 pathway and confounds attempts to investigate whether the blocking action of SOCS3 on neurite growth is mediated by blocking of STAT3 signaling or blocking of other pathways.

We have not examined the possibility that SOCS3 impairs the survival of rat DRG neurons (Yadav et al., 2005) but do not believe that increased cell death alone could explain the inhibition of neurite growth by SOCS3.

\section{Effects of endogenous SOCS3}

mSOCS3 acts as a dominant-negative reagent, blocking the actions of endogenous SOCS3 (Sasaki et al., 2000). Consequences of this action are to inhibit ERK signaling (Fig. 2) and, presumably, to enhance STAT3 signaling.

Observations that mSOCS3 significantly enhances neurite outgrowth in vitro, at least from neurons that have not been conditioned, demonstrate that endogenous SOCS3 exercises some restraint on neurite growth. The relatively small magnitude of the effect of mSOCS3 may not represent accurately the function of endogenous SOCS3 but rather may be related to the timing of mSOCS3 synthesis and critical events in the induction of neurite growth. Observations of a strong propensity for axonal growth in vivo and in axotomized DRG neurons (Richardson and Issa, 1984; Neumann and Woolf, 1999) with at least 10 times normal concentrations of endogenous SOCS3 mRNA (Fig. 1) suggest that the influence of endogenous SOCS3 on axonal growth in these neurons is limited. However, no information is available on the concentration of SOCS3 protein as opposed to mRNA in neurons nor to the distribution of SOCS3 among neurons. Also, it remains possible that SOCS3 contributes to the inability of many CNS neurons to mount an effective regenerative response. The demonstration that endogenous and exogenous SOCS3 each has an inhibitory effect on axonal growth warrants further investigation into the molecular mechanisms of this morphological action and into therapeutic manipulation of the STAT3 and SOCS3 signaling pathways.

\section{References}

Banerjee A, Banks AS, Nawijn MC, Chen XP, Rothman PB (2002) Cutting edge: suppressor of cytokine signaling 3 inhibits activation of NFATp. J Immunol 168:4277-4281.

Bomze HM, Bulsara KR, Iskandar BJ, Caroni P, Skene JH (2001) Spinal axon regeneration evoked by replacing two growth cone proteins in adult neurons. Nat Neurosci 4:38-43.

Bonilla IE, Tanabe K, Strittmatter SM (2002) Small proline-rich repeat protein $1 \mathrm{~A}$ is expressed by axotomized neurons and promotes axonal outgrowth. J Neurosci 22:1303-1315.

Cacalano NA, Sanden D, Johnston JA (2001) Tyrosine-phosphorylated SOCS-3 inhibits STAT activation but binds to p120 RasGAP and activates Ras. Nat Cell Biol 3:460-465.

Cafferty WB, Gardiner NJ, Gavazzi I, Powell J, McMahon SB, Heath JK, Munson J, Cohen J, Thompson SW (2001) Leukemia inhibitory factor determines the growth status of injured adult sensory neurons. J Neurosci 21:7161-7170.

Cafferty WB, Gardiner NJ, Das P, Qiu J, McMahon SB, Thompson SW (2004) Conditioning injury-induced spinal axon regeneration fails in interleukin-6 knock-out mice. J Neurosci 24:4432-4443.

Croker BA, Metcalf D, Robb L, Wei W, Mifsud S, DiRago L, Cluse LA, Sutherland KD, Hartley L, Williams E, Zhang JG, Hilton DJ, Nicola NA, Alexander WS, Roberts AW (2004) SOCS3 is a critical physiological negative regulator of G-CSF signaling and emergency granulopoiesis. Immunity 20:153-165. 
Dauer DJ, Ferraro B, Song L, Yu B, Mora L, Buettner R, Enkemann S, Jove R, Haura EB (2005) Stat3 regulates genes common to both wound healing and cancer. Oncogene 24:3397-3408.

Fischer P, Lehmann U, Sobota RM, Schmitz J, Niemand C, Linnemann S, Haan S, Behrmann I, Yoshimura A, Johnston JA, Muller-Newen G, Heinrich PC, Schaper F (2004) The role of the inhibitors of interleukin-6 signal transduction SHP2 and SOCS3 for desensitization of interleukin-6 signalling. Biochem J 378:449-460.

Galiano M, Liu ZQ, Kalla R, Bohatschek M, Koppius A, Gschwendtner A, Xu S, Werner A, Kloss CU, Jones LL, Bluethmann H, Raivich G (2001) Interleukin-6 (IL6) and cellular response to facial nerve injury: effects on lymphocyte recruitment, early microglial activation and axonal outgrowth in IL6-deficient mice. Eur J Neurosci 14:327-341.

Greenhalgh CJ, Alexander WS (2004) Suppressors of cytokine signalling and regulation of growth hormone action. Growth Horm IGF Res 14:200-206.

Heinrich PC, Behrmann I, Haan S, Hermanns HM, Muller-Newen G, Schaper F (2003) Principles of interleukin (IL)-6-type cytokine signalling and its regulation. Biochem J 374:1-20.

Ihara S, Nakajima K, Fukoka T, Hibi M, Nagata S, Hirano T, Fukui Y (1997) Dual control of neurite outgrowth by STAT3 and MAP kinase in PC12 cells stimulated with interleukin-6. EMBO J 17:5345-5352.

Kobayashi NR, Fan D-P, Giehl KM, Bedard AM, Wiegand SJ, Tetzlaff W (1997) BDNF and NT-4/5 prevent atrophy of rat rubrospinal neurons after cervical axotomy, stimulate GAP- 43 and T $\alpha 1$-tubulin mRNA expression, and promote axonal regeneration. J Neurosci 17:9583-9595.

Li W, Lee J, Vikis HG, Lee SH, Liu G, Aurandt J, Shen TL, Fearon ER, Guan JL, Han M, Rao Y, Hong K, Guan KL (2004) Activation of FAK and Src are receptor-proximal events required for netrin signaling. Nat Neurosci 7:1213-1221.

Liu E, Cote JF, Vuori K (2003) Negative regulation of FAK signaling by SOCS proteins. EMBO J 22:5036-5046.

Liu RY, Snider WD (2001) Different signaling pathways mediate regenerative versus developmental sensory axon growth. J Neurosci 21:RC164(1-5).

Matsuda T, Nakamura T, Nakao K, Arai T, Katsuki M, Heike T, Yokota T (1999) STAT3 activation is sufficient to maintain an undifferentiated state of mouse embryonic stem cells. EMBO J 18:4261-4269.

Milocco LH, Haslam JA, Rosen J, Seidel HM (1999) Design of conditionally active STATs: insights into STAT activation and gene regulatory function. Mol Cell Biol 19:2913-2920.

Mori H, Hanada R, Hanada T, Aki D, Mashima R, Nishinakamura H, Torisu T, Chien KR, Yasukawa H, Yoshimura A (2004) Socs3 deficiency in the brain elevates leptin sensitivity and confers resistance to diet-induced obesity. Nat Med 10:739-743.

Murphy PG, Grondin J, Altares M, Richardson PM (1995) Induction of interleukin-6 in axotomized sensory neurons. J Neurosci 15:5130-5138.

Neumann S, WoolfCJ (1999) Regeneration of dorsal column fibres into and beyond the lesion site following adult spinal cord injury. Neuron 23:83-91.

Ng YP, He W, Ip NY (2003) Leukemia inhibitory factor receptor signaling negatively modulates nerve growth factor-induced neurite outgrowth in PC12 cells and sympathetic neurons. J Biol Chem 278:38731-38739.

Pradervand S, Yasukawa H, Muller OG, Kjekshus H, Nakamura T, St Amand TR, Yajima T, Matsumura K, Duplain H, Iwatate M, Woodard S, Pedrazzini T, Ross J, Firsov D, Rossier BC, Hoshijima M, Chien KR (2004) Small proline-rich protein $1 \mathrm{~A}$ is a gp130 pathway- and stress-inducible cardioprotective protein. EMBO J 23:4517-4525.

Ren XR, Ming GL, Xie Y, Hong Y, Sun DM, Zhao ZQ, Feng Z, Wang Q, Shim S, Chen ZF, Song HJ, Mei L, Xiong WC (2004) Focal adhesion kinase in netrin-1 signaling. Nat Neurosci 7:1204-1212.

Richardson PM, Issa VMK (1984) Peripheral injury enhances central regeneration of primary sensory neurons. Nature 309:791-793.

Roberts AW, Robb L, Rakar S, Hartley L, Cluse L, Nicola NA, Metcalf D, Hilton DJ, Alexander WS (2001) Placental defects and embryonic lethality in mice lacking suppressor of cytokine signaling 3. Proc Natl Acad Sci USA 98:9324-9329.

Rui L, Yuan M, Frantz D, Shoelson S, White MF (2002) SOCS-1 and SOCS-3 block insulin signaling by ubiquitin-mediated degradation of IRS1 and IRS2. J Biol Chem 277:42394-42398.

Ruitenberg MJ, Plant GW, Christensen CL, Blits B, Niclou SP, Harvey AR, Boer GJ, Verhaagen J (2002) Viral vector-mediated gene expression in olfactory ensheathing glia implants in the lesioned rat spinal cord. Gene Ther 9:135-146.

Sasaki A, Yasukawa H, Shouda T, Kitamura T, Dikic I, Yoshimura A (2000) CIS3/SOCS-3 suppresses erythropoietin (EPO) signaling by binding the EPO receptor and JAK2. J Biol Chem 275:29338-29347.

Sitko JC, Guevara CI, Cacalano NA (2004) Tyrosine-phosphorylated SOCS3 interacts with the Nck and Crk-L adapter proteins and regulates Nck activation. J Biol Chem 279:37662-37669.

Smith DS, Skene JH (1997) A transcription-dependent switch controls competence of adult neurons for distinct modes of axon growth. J Neurosci 17:646-658.

Ueki K, Kondo T, Kahn CR (2004) Suppressor of cytokine signaling 1 (SOCS-1) and SOCS-3 cause insulin resistance through inhibition of tyrosine phosphorylation of insulin receptor substrate proteins by discrete mechanisms. Mol Cell Biol 24:5434-5446.

Wu YY, Bradshaw RA (2000) Activation of the stat3 signaling pathway is required for differentiation by interleukin-6 in PC12-E2 cells. J Biol Chem 275:2147-2156.

Yadav A, Kalita A, Dhillon S, Banerjee K (2005) JAK/STAT3 pathway is involved in survival of neurons in response to insulin-like growth factor and negatively regulated by suppressor of cytokine signaling-3. J Biol Chem 280:31830-31840.

Yasukawa H, Ohishi M, Mori H, Murakami M, Chinen T, Aki D, Hanada T, Takeda K, Akira S, Hoshijima M, Hirano T, Chien KR, Yoshimura A (2003) IL-6 induces an anti-inflammatory response in the absence of SOCS3 in macrophages. Nat Immunol 4:551-556.

Zhou FQ, Zhou J, Dedhar S, Wu YH, Snider WD (2004) NGF-induced axon growth is mediated by localized inactivation of GSK-3beta and functions of the microtubule plus end binding protein APC. Neuron 42:897-912. 